\title{
Determination of Oxygen Permeability in Soft Contact Lenses Using a Polarographic Method: Estimation of Relevant Physiological Parameters
}

\author{
J. M. Gonzalez-Meijome, ${ }^{\dagger}$ V. Compañ-Moreno, ${ }^{*, \hbar}$ and E. Riande ${ }^{\S}$ \\ Dpto. Física (Optometria). Universidade do Minho. Campus de Gualtar. 4710-057 - Braga. (Portugal), Dpto. \\ Termodinámica Aplicada. ETSII. Universidad Politécnica de Valencia. 46022-Valencia. (Spain), and Instituto \\ de Polímeros. Consejo Superior de Investigaciones Científicas. 28006, Madrid. (Spain)
}

\begin{abstract}
This work reports the apparent oxygen transmissibility $D k / t)_{\text {app }}$ of four silicone hydrogel $(\mathrm{Si}-\mathrm{Hy})$ contact lenses (CLs). A method is described that allows the estimation of the oxygen tension at the lens-cornea interface for closed- and open-eyelids situations combining the instrument oxygen transmissibility (IOT) and corneal parameters such as corneal thickness, corneal permeability, and oxygen flux across the cornea. From these results, the biological oxygen apparent transmissibility (BOAT), equivalent oxygen percentage (EOP), partial pressure, $\left(p_{\mathrm{tc}}\right)$, of oxygen at the cornea-CL interface and oxygen flux, $\left(j_{\mathrm{c}}\right)$, were also obtained. This method allows the evaluation of the physiological environment under the lens using the formulations described in previous studies. The oxygen performance of four $\mathrm{Si}-\mathrm{Hy}$ materials was evaluated using a polarographic cell coupled to a permeometer. Measurements of the apparent transmissibility, $(D k / t)_{\text {app }}$, and permeability, $D k$, were performed in stacks containing from 1 to $n$ repeated lenses and, from the values obtained, the error involved in the measurements in single samples was estimated. It was found that the values of $(D k / t)_{\text {app }}$ and $D k$ obtained following the two different procedures (stack method and measurement of single sample) were significantly different from the nominal values given by the manufacturer, particularly for some samples. However, the impact of these differences on the values of the other physiologically relevant parameters $\left(\mathrm{BOAT}, \mathrm{EOP}, p_{\mathrm{tc}}\right.$, and $j_{\mathrm{c}}$ ) was not significant. Furthermore, these parameters were similar for the four lenses in spite of the different $(D k / t)_{\text {app }}$ measured. The relationships of $(D k / t)_{\text {app }}$ with the remaining physiological parameters were calculated and graphically represented for open and closed-eyelid conditions.
\end{abstract}

\section{Introduction}

Since the first attempts to prescribe overnight contact lens (CL) wear during the $1980 \mathrm{~s}$, the increase of the oxygen permeability of CL materials has been a challenge for scientists involved in CL engineering. Corneal swelling, ${ }^{1}$ limbal redness, ${ }^{2}$ epithelial thinning, ${ }^{3}$ mycrocists ${ }^{4,5}$ are just some of the complications attributed to corneal oxygen depletion during CL wear. Under normal open-eyelid conditions, the cornea at sea level requires a minimum oxygen supply of 5 to $7.5 \mu \mathrm{L} \cdot \mathrm{cm}^{-2} \cdot$ hour $^{-1}$.6,7 Holden and Mertz $^{8}$ predicted that lenses under daily wear conditions should provide a minimum $D k / t$ of 34 barrer/cm, or an equivalent oxygen percentage (EOP) of $9.9 \%$, whereas this value would increase up to 87 barrer/cm $(\mathrm{EOP}=17.9 \%)$ to prevent corneal hypoxia and thus limit corneal edema to physiological levels $(<4 \%)$. However, more recent estimates made by Harvitt and Bonanno, ${ }^{9}$ utilizing the metabolic requirements of the cornea under hypoxic conditions, suggest the necessity of providing the cornea with higher levels of oxygenation to avoid hypoxia through the whole cornea under overnight CL wear. In this case, a minimum $D k / t$ of 125 barrer/ $\mathrm{cm}$ would be necessary. Recent studies conducted by Compan et al. ${ }^{10}$ reported that CLs with oxygen transmissibility higher than 100 barrer/cm provide the lens - cornea interface with an oxygen tension that reduces the concentration gradient, though the oxygen flux into the cornea increases due to the higher $D k$ / $t$. According to these studies, increasing the value of $D k / t$ above

* To whom correspondence should be addressed. E-mail: vicommo@ter.upv.es.

†niversidade do Minho. Campus de Gualtar.

Universidad Politécnica de Valencia.

$\S$ Instituto de Polímeros.
70 barrer/cm will have a minor impact on the oxygen flux onto the cornea, even under overnight wear. Brennan ${ }^{11}$ reported that values of $D k / t$ of 15 and 50 barrer/cm will be enough to satisfy $96 \%$ of the cornea's normal oxygen consumption in daily and overnight wear.

Nowadays, new polymers containing highly permeable siloxane moieties are available, which significantly improve oxygen permeation performance. Some of these materials have been worn under continuous wear for periods up to 30 days without relevant ocular complications. ${ }^{12-17}$ As a consequence, these materials also offer the possibility of being continuously worn for therapeutic use. ${ }^{18-21}$

Four different procedures have been used to determine the oxygen transmissibility and permeability coefficients of CLs. Three of these procedures use a Clark oxygen electrode covered by the lens directly, ${ }^{22}$ or separated by a thin Teflon membrane of known oxygen transmissibility, to measure the oxygen flux through the lenses. ${ }^{23,24}$ The first method was developed for hydrogel lenses that are swollen in the electrolyte required for the electrochemical reaction to take place on the electrode. The second method is similar to the first one but adapted for rigid hydrophobic CLs. In this case, a thin piece of cigarette paper soaked in an electrolyte solution is sandwiched between the hydrophobic lens and the electrode to establish the electrolytic contact between the lens and the electrode..$^{25,26}$ The third method, which can be used for hydrogels as well as for rigid lenses, contains an electrolyte solution between the Teflon membrane and the electrode. ${ }^{23,27}$ The fourth method uses dual chambers separated by the membrane whose $D k / t$ is to be measured. Oxygen introduced into one of the chambers diffuses through 
the lens from the chamber with the higher partial pressure of oxygen to the second chamber fitted with an oxygen-consuming electrode. ${ }^{28}$

The electrochemical technique described by Aiba et al. ${ }^{29}$ for polymeric membranes has often been used for the determination of the oxygen permeability coefficient of hydrogel CLs placed directly on the electrode. This technique, known as the polarographic method, allows the determination of the oxygen flux through the lens from the measurement of an electric current in a potentiometer. However, this technique is adversely affected by the so-called boundary-layer effect that leads to the underestimation the oxygen transmissibility. Actually, the resistance of the boundary layers flanking the lens causes a decrease in the oxygen partial pressure at the side of the lens in contact with the high-pressure compartment accompanied by an increase at the side of the lens facing the electrode. This effect leads to a diminution of oxygen transport across the lens. This problem can be overcome by measuring material samples of different thickness or stacking several lenses of known thickness. ${ }^{30}$ Young and Benjamin ${ }^{31}$ used this approach in powered CLs made of lotrafilcon A and balafilcon A. The other major concern with the polarographic technique is the so-called edge effect that affects measurements when the area at both sides of the membrane (i.e., CL) is not the same. This is the case for powered CLs or when oxygen transport is not perpendicular to the lens surface. A small lateral diffusion could happen, and as a consequence the actual area of the membrane exposed to the atmosphere would by higher than that assumed. This effect that conducts to an overestimation of the oxygen transmissibility can be avoided by adjusting the actual surface area of the CL exposed to the cathode of the cell and by incorporating different correction factors already available in some commercial devices.

Gas-gas and coulometric techniques can be used to measure gas transport in lenses, though they work in different way. Thus, whereas in the former technique the lens is the only area that communicates two different chambers (some authors call this procedure the dual-chamber technique), in the coulometric method one chamber is saturated with oxygen and the other, in contact with the cathode, is saturated with nitrogen. The amount of oxygen detected at the second chamber serves to evaluate the diffusion of oxygen through the lens. Though both techniques are relatively free from boundary-layer effects (only the anterior boundary layer is present in the measurements of oxygen transport across hydrophilic samples with the coulometric technique), the edge effect still persists in the polarographic method. ${ }^{32}$ Gas-to-gas technique is not suitable to measure oxygen transport in swollen hydrophilic samples.

The polarographic method is not recommended by ISO standards (ISO $9913-1)^{33}$ to measure permeability coefficients of 100 barrers or higher in CLs. On the other hand, the coulometric method is more suitable for lenses with $D k>70$ barrer. ${ }^{34}$ Despite this, both methods are currently used to obtain $D k / t$ and $D k$ coefficients of modern high- $D k$ (>100 barrer) $\mathrm{Si}-$ Hy CLs, with satisfactory results, according to the nominal values given by the manufacturers. ${ }^{10,30,35}$

Morgan et al. ${ }^{34}$ evaluated the oxygen transmissibility and permeability of lotrafilcon A (Focus Night \& Day, CIBA Vision; Duluth, GA), obtaining some discrepancies between the coulometric (in the liquid-gas configuration) and polarographic techniques for lotrafilcon $\mathrm{A}$ and other RGP materials with permeability coefficients higher than 70 barrer. The discrepancies not only affect the mean values but also the standard error, which is larger with the polarographic technique. Alvord et al. ${ }^{35}$ compared permeation results from the liquid-to-gas configuration with those from the gas-to-gas configuration of the coulometric technique for the lotrafilcon A material. The gas-to-gas configuration used gave higher $D k$ values due to partial lens desiccation, presumably as a consequence of the high permeability of dry silicon moieties. Morgan et al. ${ }^{34}$ and Alvord et al. ${ }^{35}$ found similar $D k$ values for lotrafilcon A using the liquidto-gas method in the coulometric device, the values obtained being $150 \pm 4$ and $155 \pm 5$ barrers, respectively. Using the polarographic technique, Compañ et al. ${ }^{30}$ found that the oxygen permeability of lotrafilcon A is $141 \pm 5$ barrer, proving that even in high- $D k$ SCLs accurate and repeatable measurements can be obtained with this technique. Recent work of Young and Benjamin ${ }^{31}$ and Compañ et al. ${ }^{10,30}$ also provided a term of comparison for balafilcon A (Purevision, Bausch \& Lomb; Rochester, NY). These authors reported values of transmissibility lying in the range $102-111$ barrer, being the mean value $107 \pm 4$ barrers. Chhadra et al., ${ }^{36}$ using a novel polarographic apparatus with only a single soft contact lens (SCL), measured oxygen permeabilities of hypertransmissible SCLs ranging from 9 to 180 barrer, in excellent agreement with those claimed by commercial manufacturers. For example, for balafilcon A (Purevision, Bausch \& Lomb; Rochester, NY) and lotrafilcon A of thicknesses $94 \mu \mathrm{m}$ and $90 \mu \mathrm{m}$, respectively, the permeability coefficients obtained were 108 and 181 barrers, respectively. These results are similar to those reported by Compañ et al. ${ }^{10,30}$ for these materials.

Recently, new materials are being launched to the marketplace based on the $\mathrm{Si}-\mathrm{Hy}$ technology. Although they are not intended to be worn on an extended or continuous wear schedule, their oxygen performance as labeled by their manufacturers seems to be very close to satisfying the more stringent criteria presently required. According to the different studies quoted above, it is not clear which of the measurement methods should be used to estimate the performance of high oxygen transmissibility lenses.

The purpose of this study was to measure the apparent oxygen transmissibility $(D k / t)_{\text {app }}$ of four $\mathrm{Si}-\mathrm{Hy}$ CLs using the polarographic technique with edge effect correction. Also the possibility of boundary-layer corrections by stacking several lenses of the same material is discussed. The transmissibility of single samples was also obtained. From the measurements, the values of BOAT, EOP, $p_{\mathrm{tc}}$, and $j_{\mathrm{c}}$ under open- and closed-eyelid conditions were derived and compared with those derived from nominal $D k / t$ values given by the manufacturers.

\section{Materials and Methods}

2.1. Contact Lenses. Four $\mathrm{Si}-\mathrm{Hy}$ CL materials currently available in the world market have been used in this study including Air Optix Night \& Day (lotrafilcon A-24\%) and Air Optix (lotrafilcon B-33\%) from CIBA Vision Corporation, Duluth, GA; Acuvue Oasys (senofilcon A-38\%) from Johnson \& Johnson Vision Care, Jacksonville, FL; and PureVision (balafilcon A-36\%) from Bausch \& Lomb, Inc., Rochester, NY. Technical details of the $\mathrm{Si}-\mathrm{Hy}$ materials are displayed in Table 1.

2.2. Experimental Device. The amount of oxygen passing through the CL was computed from the measurement of the electric current generated by the reduction of oxygen at the cathode of a modified polarographic electrode (Rehder Development Co.; Castro Valley, CA) when the gold cathode is maintained at $-0.75 \mathrm{~V}$ with respect to the silver anode coupled to a Model 201T $\mathrm{O}_{2}$ Permeometer (Createch; Albany, CA). To mimic ocular conditions during measurements, the system was set using a thermostat at $35 \pm 1{ }^{\circ} \mathrm{C}$.

The electrodes were wetted by a drop of a buffer solution ${ }^{37}$ $(0.9 \% \mathrm{NaCl}$ in water +1 drop of borax $)$ to keep the $\mathrm{pH}$ in the 
Table 1. Technical Details of $\mathrm{Si}-\mathrm{Hy}$ CLs Used in the Study

\begin{tabular}{|c|c|c|c|c|}
\hline & $\begin{array}{l}\text { Air Optix } \\
\text { Night\&Day }\end{array}$ & $\begin{array}{l}\text { Acuvue } \\
\text { Oasys }\end{array}$ & Air Optix & Purevision \\
\hline material & lotrafilcon A & senofilcon A & lotrafilcon B & balafilcon A \\
\hline$D k$ (barrer) & 140 & 103 & 110 & 99 \\
\hline$t_{\mathrm{c}}(\mathrm{mm} @-3.00)$ & 80 & 70 & 80 & 90 \\
\hline$t_{\mathrm{c}}$ (measured)* & $85 \pm 6$ & $72 \pm 2$ & $85 \pm 4$ & $96 \pm 4$ \\
\hline$D k / t$ (barrer/cm) & 175 & 147 & 138 & 110 \\
\hline $\mathrm{H}_{2} \mathrm{O}(\%)$ & $24 \%$ & $38 \%$ & $33 \%$ & $36 \%$ \\
\hline FDA & I & I & I & III \\
\hline power (D) & -3.00 & 3.00 & -3.00 & 3.00 \\
\hline diameter (mm) & 13.8 & 14.6 & 14.2 & 14 \\
\hline base curve (mm) & 8.6 & 8.7 & 8.6 & 8.6 \\
\hline $\begin{array}{l}\text { schedule/ } \\
\text { replacement }\end{array}$ & CW/monthly & DW/monthly & DW/monthly & CW/monthly \\
\hline
\end{tabular}

interval of 6.0-7.4. The sample was taken out of a reservoir containing the same buffer solution and placed onto the surface of the electrodes. The hydrogel was fixed gently by pressing a hollow cylinder with a tightening $\mathrm{O}$ ring toward the polymer and the electrodes. Finally, about $0.5 \mathrm{~cm}^{3}$ of the buffer solution were poured through the hollow cylinder on the hydrogel, and the system was ready for the measurements of the electric current. Figure 1shows a graphical representation of the elements within the measuring cell.

Because the solution and the hydrogel were saturated with atmospheric oxygen, before each experiment and with the current turned on, nitrogen saturated with water vapor was bubbled through the saline solution in the hollow cylinder until the residual current decreased to nearly zero. When all of the oxygen was practically eliminated, moist air at $1 \mathrm{~atm}$ of pressure was bubbled into the top solution, and the increase of the electric current with time was recorded until a stationary state was reached. The system was monitored using a thermostat during the permeation experiments.

2.2. Measurements of Apparent Oxygen Transmissibility: Theoretical Background. In the apparatus used in this study, the oxygen permeates from one side of the lens where the partial pressure of the gas is kept constant $\left(p=p_{\mathrm{t}}=155\right.$ $\mathrm{mmHg})$ ) to the other side facing the cathode of the polarographic cell where the partial pressure is $p_{0}=\sim 0$. In steady-state conditions, the flux of oxygen across CLs is given by

$$
J_{\mathrm{x}}=P \frac{p_{\mathrm{t}}-p_{0}}{t_{\mathrm{av}}}=P \frac{p_{\mathrm{t}}}{t_{\mathrm{av}}}
$$

where $P$ is the permeability coefficient, and $p_{\mathrm{t}}$ and $p_{0}$ are, respectively, the pressures of oxygen flanking the lens in the permeometer. The permeability coefficient can be expressed in terms of the diffusion, $D$, and solubility, $k$, coefficients as

$$
P=k D
$$

By combining eqs 1 and 2 and taking into account that the reduction of a mole of oxygen consumes $n F$ Coulombs, where $F$ is Faraday's constant and $n=4$, the apparent oxygen transmissibility measured by the instrument (IOT) is related to the total current diffusion in the steady state $(I)$ by the following equation,

$$
\left(\frac{D k}{t_{\mathrm{av}}}\right)_{\mathrm{app}}=\frac{I}{n F A \Delta p}=B I
$$

where $\Delta p$ is the oxygen partial pressure difference across the lens $[=15.5 \mathrm{cmHg}$ (at sea level)] and $A$ is the permeation area. For our cell, $B=(n F A \Delta p)^{-1}=0.02929\left\{\mathrm{~cm}^{3} \mathrm{O}_{2}\left(S T_{\mathrm{p}}\right)\right\} /\left\{\mathrm{cm}^{2}\right.$. $\mathrm{s}^{\bullet} \mathrm{cmHg}$ \} Therefore, $B$ is the cell constant. The harmonic average thickness of the CLs $\left(t_{\mathrm{av}}\right)$ was calculated from five measurements in five regions of the permeation zone of the lens within a 2.5 $\mathrm{mm}$ radius, corresponding to the active part of the lens in contact with the cathode.

From eqs 2 and 3, the apparent permeability is the value of the IOT times the harmonic mean thickness of the polymer, $t_{\mathrm{av}}$ :

$$
P=D k=\frac{I t_{\mathrm{av}}}{n F A \Delta p}=B I t_{\mathrm{av}}
$$

Under non-steady-state conditions, gas transport across lenses is governed by Fick's second law. By integrating this equation using appropriate boundary conditions, the amount of electric current spent from 0 to time $t$ in the oxidation of oxygen flowing across the membrane is given by ${ }^{38}$

$$
\begin{array}{r}
Q(t)=\frac{n F A D c_{0}}{t_{\mathrm{av}}}\left[\left(t-\frac{t_{\mathrm{av}}{ }^{2}}{6 D}\right)-\frac{2 t_{\mathrm{av}}^{2}}{D \pi^{2}} \sum_{n=1}^{\infty}(-1)^{n} \times\right. \\
\left.\exp \left(-\frac{n^{2} \pi^{2} D t}{t_{\mathrm{av}}}\right)\right]
\end{array}
$$

In steady-state conditions $(t \rightarrow \infty)$, the current intensity can be written as

$$
I(\infty)=\lim _{t \rightarrow \infty} \frac{\mathrm{d} Q(t)}{\mathrm{d} t}=\frac{n F A D c_{0}}{t_{\mathrm{av}}}
$$

By assuming that Henry's law holds, $c_{0}=k p_{\mathrm{t}}$, where $p_{\mathrm{t}}$ is the pressure of gas at the surface of the membrane facing the high-pressure side in the experimental device, then eq 6 becomes

$$
I(\infty)=\frac{n F A P}{t_{\mathrm{av}}} p_{\mathrm{t}}
$$

In steady-state conditions, eq 11 becomes

$$
Q(t)=n F A P \frac{p_{\mathrm{t}}}{t_{\mathrm{av}}}\left(t-\frac{t_{\mathrm{av}}^{2}}{6 D}\right)=I_{\infty}\left(t-\frac{t_{\mathrm{av}}^{2}}{6 D}\right)
$$

The apparent oxygen diffusion coefficients can be determined from eq 8 as

$$
D=\frac{t_{\mathrm{av}}{ }^{2} I_{\infty}}{6\left(I_{\infty} t-Q(t)\right)}
$$

By integrating the curves $I(t)$ versus $t, Q(t)$ can be calculated. The curve $Q(t)$ versus $t$ presents a transitory process at short times followed by a steady-state process where the time dependence of $Q(t)$ is a straight line described by eq 8 . The straight line intersects the abscissa axis at a time $\theta$, called the lag time. Then the diffusion coefficient can be obtained by the procedure suggested by Barrer

$$
D=\frac{t_{\mathrm{av}}{ }^{2}}{6 \theta}
$$

This method described by Aiba et al. ${ }^{29}$ is an alternative to eq 9 to calculate the apparent diffusion coefficient.

2.3. Oxygen Permeability Determination $(D k)$. Stack Method. By measuring $I(t)$ as a function of time the values of both the permeability and diffusion coefficients can be readily 


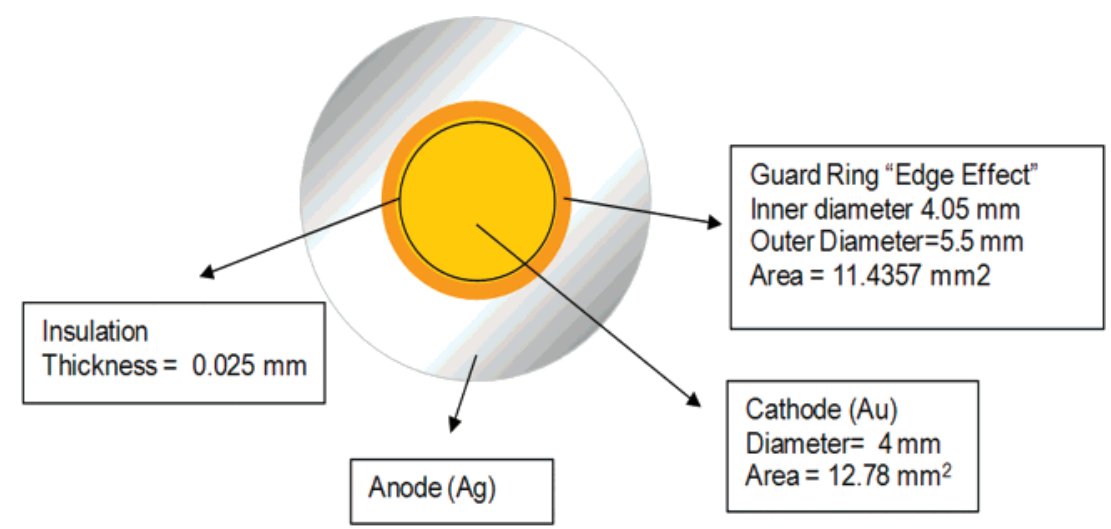

Figure 1. Representation of the measurement cell in the Rehder Permeometer.

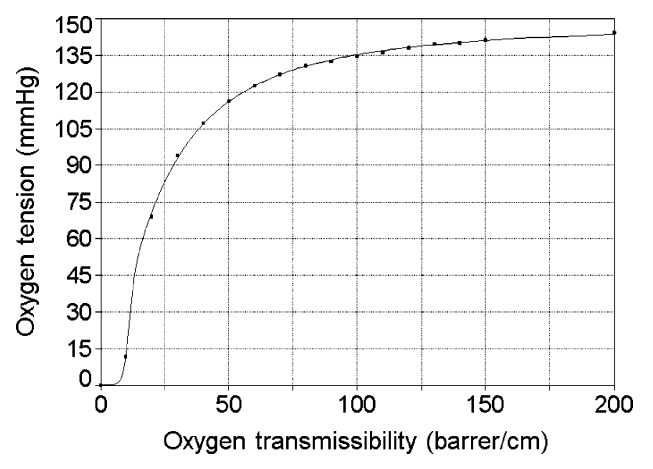

Figure 2. Model predicting the oxygen tension at the cornea-CL interface as a function of the measured $D k / t\left(D k / t_{\text {app }}\right)$ under open-eyelid conditions (from the experimental values obtained by Compañ et al. 2004).

determined, respectively, by means of eq 4 and 9 . It should be pointed out, however, that as a consequence of the liquidmembrane interfaces, the partial pressure difference between the two faces of the membrane could differ slightly from $p_{\mathrm{t}}-$ $p_{0}$. On the other hand, the diffusion resistance of the solution layer separating the membrane from the electrode has not been considered, so that the permeation measurements embody the assembly comprising the membrane and the two thin liquid solution layers flanking it. For this reason, the results obtained for $P$ and $D$ with the experimental device used in this study are apparent values. An approach to get rid of liquid layer effects, and to obtain true values of oxygen permeability, is to carry out the measurements in a stack of $n$ CLs slightly pressed so that the liquid-layer thickness between every two lenses in the stack is negligible with regard to that of single lenses. By measuring the steady-state current for stacks with different number of lenses and taking into account eq 4 one obtains,

$$
\frac{4 F A \Delta p}{I_{\infty}}=\frac{t_{\mathrm{av}}}{D k}+\frac{t}{D_{0} k_{0}}
$$

where $t_{\mathrm{av}}$ and $D k$, are, respectively, the thickness and the permeability coefficients of $n$ cumulative lenses in the stack, and the second term of the right-hand side of eq 11 is the resistance of the liquid layers flanking the stack. The plot of $4 F p_{t} A / I_{\infty, i}$ versus $t_{\mathrm{av}, i}$ should roughly give a straight line from whose slope and ordinate in the origin the reciprocal of the permeability coefficient and the resistance of the liquid layers are obtained, respectively.

2.4. Biological Oxygen Apparent Transmissibility (BOAT). The following sections will be devoted to describe the estimation of BOAT, EOP, and the oxygen flux reaching the cornea $\left(j_{\mathrm{c}}\right)$. A critical point in these calculations is the need of knowing the partial pressure of oxygen at the cornea-CL interface $\left(p_{\mathrm{tc}}\right)$ under

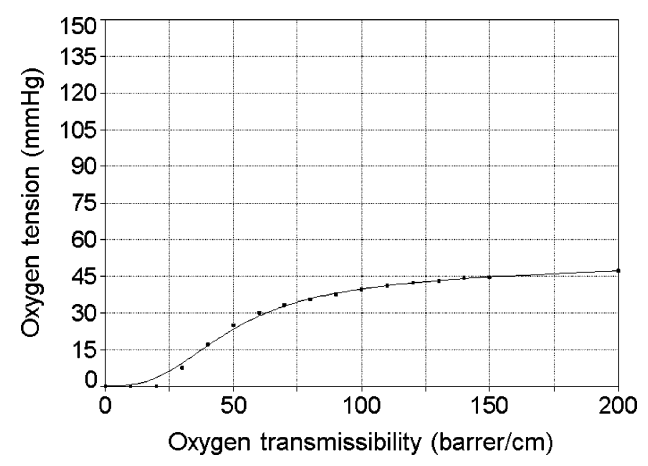

Figure 3. Model predicting the oxygen tension at the cornea-CL interface as a function of the measured $D k / t$ value $\left(D k / t_{\text {app }}\right)$ under open- and closedeye conditions (from the experimental values obtained by Compañ et al. 2004).

open- and closed-eyelid conditions. By relating the flux of oxygen across the lenses obtained form the IOT with the flux reaching the cornea, one finds

$$
j_{\mathrm{c}}=\frac{D k}{t_{\mathrm{av}}} p\left(\frac{p-p_{\mathrm{tc}}}{p}\right)
$$

where $p$ is equal to ca. $155 \mathrm{mmHg}\left(21 \% \mathrm{O}_{2}\right.$ at the variable atmospheric pressure at sea level). Fatt and Ruben ${ }^{39}$ established the BOAT concept that combines the physiological parameter $p_{\text {tc }}$ and the instrument oxygen transmissibility of the lens expressed by IOT. The BOAT parameter is given by

$$
[\mathrm{BOAT}]=\frac{D k}{t_{\mathrm{av}}}\left(\frac{p-p_{\mathrm{tc}}}{p}\right)
$$

Then, the flow of oxygen onto the cornea can directly be obtained from the BOAT using the following expression

$$
j_{\mathrm{c}}=[\mathrm{BOAT}] p
$$

The estimation of the BOAT requires the evaluation of the oxygen tension at the lens-cornea interface, $p_{\mathrm{tc}}$, and the IOT of the lens. Details are available in Appendix A. Compañ et al. ${ }^{10}$ have reported statistical relationships between $p_{\text {tc }}$ and for values of $(D k / t)_{\text {app }}$ ranging from 0 to 300 barrer $/ \mathrm{cm}$.

2.5. Oxygen Tension at the Lens-Cornea Interface $\left(p_{\text {tc }}\right)$. The value of the parameter $p_{\mathrm{tc}}$ is essential to obtain the BOAT, EOP, and the flux of oxygen onto the cornea under open- and closed-eyelid conditions. Compañ et al. ${ }^{10}$ have calculated the oxygen tension behind the CL $\left(p_{\mathrm{tc}}\right)$ as a function of instrument oxygen transmissibility $(D k / t)_{\text {app. }}$ In the present work, the equations that correlate these parameters have been obtained 


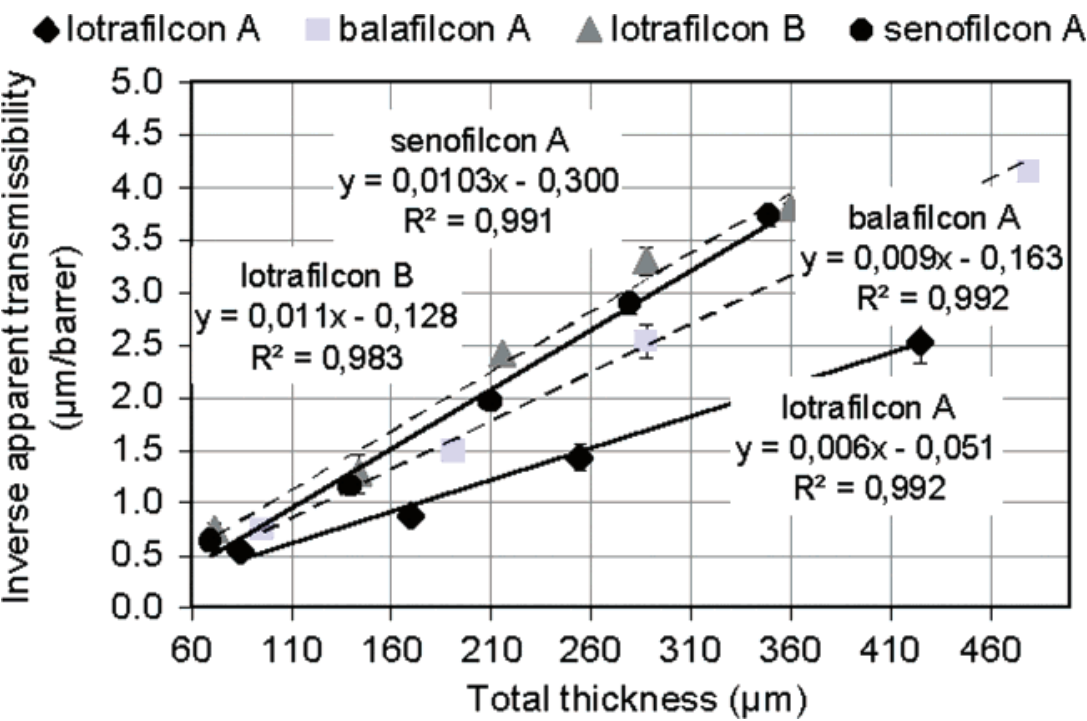

Figure 4. Inverse transmissibility vs thickness of stacked lenses for $\mathrm{Si}-\mathrm{Hy}$ materials. The average values are presented.

Table 2. Oxygen Transmissibility Obtained by Different Methods and Values Reported by the Manufacturers for Different $\mathrm{Si}-\mathrm{Hy}$ $\mathrm{CLS}^{a}$

\begin{tabular}{cllll}
\hline & lotrafilcon A & balafilcon A & lotrafilcon B & senofilcon A \\
\hline nominal $D k / t^{\mathrm{b}}$ & 175 & 110 & 138 & 147 \\
$(D k / t)_{\mathrm{app}}$ & $182 \pm 31$ & $133 \pm 18$ & $136 \pm 19$ & $157 \pm 18$ \\
$\begin{array}{c}(\mathrm{barrer} / \mathrm{cm})^{c} \\
(D k / t)_{\mathrm{app}}\end{array}$ & $186 \pm 28$ & $118 \pm 12$ & $126 \pm 19$ & $135 \pm 16$ \\
$\quad(\mathrm{barrer} / \mathrm{cm})^{d}$ & & & &
\end{tabular}

${ }^{a}$ Values $\pm \mathrm{SD}$ are the values directly measured in this study. The average and $\mathrm{SD}$ are the result of five measurements. Units are barrer/cm $=10^{-9}$ $\left(\mathrm{cm} \mathrm{mL} \mathrm{O} \mathrm{O}_{2}(\mathrm{STP})\right) /(\mathrm{mL}$ sec $\mathrm{mmHg}) .{ }^{b}$ Nominal values given by the manufacturers. ${ }^{c}$ Derived from a single measurement of $(D k / t)_{\text {app. }}$ The corresponding $D k$ result from the multiplication of this value by the average thickness of the sample. ${ }^{d}$ Derived from the slope of the plot in the stack method. The corresponding $D k / t$ is the value of $D k$ obtained from the stack divided by average thickness of the sample.

using the methodology described in the following two sections for open- and closed-eyelid conditions. For these calculations, the following key values were used: cornea oxygen consumption $\mathrm{Q} \cong 6.6 \times 10^{-5} \mathrm{~cm}^{3} \mathrm{O}_{2}\left(S T_{\mathrm{p}}\right) \cdot \mathrm{cm}^{-3} \cdot \mathrm{s}^{-1}$, corneal thickness $=0.05 \mathrm{~cm}$, and $D k=24.7$ barrer for the corneal tissue, a value reported by Fatt and Weissman. ${ }^{40}$

By combining eqs A.8, A.9, and A.10-a for day and A.10-b for night, together with A.13, a system of three equations with three unknown parameters $\left(\mathrm{B}, \mathrm{B}^{\prime}\right.$, and $\left.\mathrm{C}^{\prime}\right)$ is obtained

$$
\begin{gathered}
t_{\mathrm{c}} B-t_{\mathrm{av}} B^{\prime}-C^{\prime}=-\left(\frac{Q}{2 D k}\right)_{\mathrm{c}} t_{\mathrm{c}}^{2}-55 \\
(D k)_{\mathrm{c}} B-(D k)_{\mathrm{tc}} B^{\prime}=-Q t_{\mathrm{c}} \\
t^{\prime} B^{\prime}+C^{\prime}=155
\end{gathered}
$$

where $p_{\mathrm{k}}$ is given in $\mathrm{mmHg}$. This system permits to determine the partial pressure of oxygen at the lens - cornea interface, $p_{\text {tc }}$. For the closed-eyelid situation, eq 17 is substituted by eq A.10b.

2.5.1. Open-Eyelid Conditions. According to the calculations described in Appendix A, $p_{\mathrm{tc}}$ was obtained from $(D k / t)_{\mathrm{app}}$ measurements. The Table Curve 2D (Jandel Scientific) was used to obtain the graphic representation shown in Figure 2.

2.5.2. Closed-Eyelid Conditions. Values of $p_{\text {tc }}$ obtained using the same approach as that utilized for open-eyelid conditions are shown as a function of the transmissibility in Figure 3.
Table 3. Oxygen Permeability Values Obtained from Different Methods and Values Reported by the Manufacturers for Different $\mathrm{Si}-\mathrm{Hy} \mathbf{C L s}^{a}$

\begin{tabular}{lllll}
\hline & lotrafilcon A & balafilcon A & lotrafilcon B & senofilcon A \\
\hline${\text { nominal } D k^{\mathrm{b}}}^{\mathrm{N}}$ & 140 & 99 & 110 & 103 \\
$D k$ (barrer $^{c}$ & $155 \pm 16$ & $127 \pm 12$ & $116 \pm 11$ & $113 \pm 10$ \\
$D k$ (barrer) $^{d}$ & $158 \pm 13$ & $106 \pm 6$ & $107 \pm 11$ & $97 \pm 9$
\end{tabular}

${ }^{a}$ Values with SD $( \pm)$ are the values directly measured in the study. The average and SD are the result from five measurements. Units are barrer = $10^{-11}\left(\mathrm{~cm}^{2} / \mathrm{sec}\right)\left[\mathrm{mL} \mathrm{O}_{2}(\mathrm{STP}) /(\mathrm{mL} \times \mathrm{mmHg})\right]$ ) (Unifica con las unidades anteriores). ${ }^{b}$ Nominal values given by the manufacturers. ${ }^{c}$ Derived from a single measurement of $(D k / t)_{\text {app }}$. The corresponding $D k$ value result from the multiplication of this value by the average thickness of the sample. ${ }^{d}$ Derived from the slope in the stack method. The corresponding $D k / t$ is the value of $D k$ obtained by the stack divided by average thickness of the sample.

2.6. Equivalent Oxygen Percentage (EOP). EOP, a clinical parameter used to represent the oxygen crossing the CL that reaches the cornea, relates the corneal swelling after wearing a specific lens with the swelling obtained in experiments involving the circulation of a series of hypoxic air of known oxygen concentration over eyes fitted with swimming goggles. ${ }^{2}$ Using the equation of Turnbull et al., ${ }^{41}$ and assuming a percentage of oxygen at sea level of 20.93 and $7.425 \%$ for open- and closedeyelid conditions, respectively, the EOP can be calculated from eq 18 as,

$$
\mathrm{EOP}=\frac{p_{\mathrm{tc}} p^{\prime}}{p}
$$

where $p$ is 155 and $55 \mathrm{mmHg}$ for open- and closed-eyelid conditions, respectively, whereas $p^{\prime}$ is 20.93 and $7.425 \%$ for the same conditions. By using the appropriate values of $p$ and $p^{\prime}$ in eq 17 , the expression that relates the parameter EOP with $p_{\text {tc }}$ for open- and closed-eyelid conditions is given by

$$
\mathrm{EOP}=0.135 p_{\mathrm{tc}}
$$

Usually the EOP is given as a percentage.

2.7. Oxygen Flux to the Cornea $\left(\boldsymbol{j}_{\mathbf{c}}\right)$. Finally, the oxygen flux to the cornea can be obtained by eq 19 using $p=155$ and $55 \mathrm{mmHg}$ for the pressure partial of oxygen at the lens-air interface for open- and closed-eyelid conditions, respectively. The flux of oxygen thus obtained is usually given in $10^{-7}$ $\left[\mathrm{cm}^{3} \mathrm{O}_{2}\left(S T_{\mathrm{p}}\right) \cdot \mathrm{cm}^{-2} \cdot \mathrm{s}^{-1}\right]$ or $\mu \mathrm{l}\left(\mathrm{O}_{2}\right) \cdot \mathrm{cm}^{-2} \cdot \mathrm{h}^{-1}$ units. 

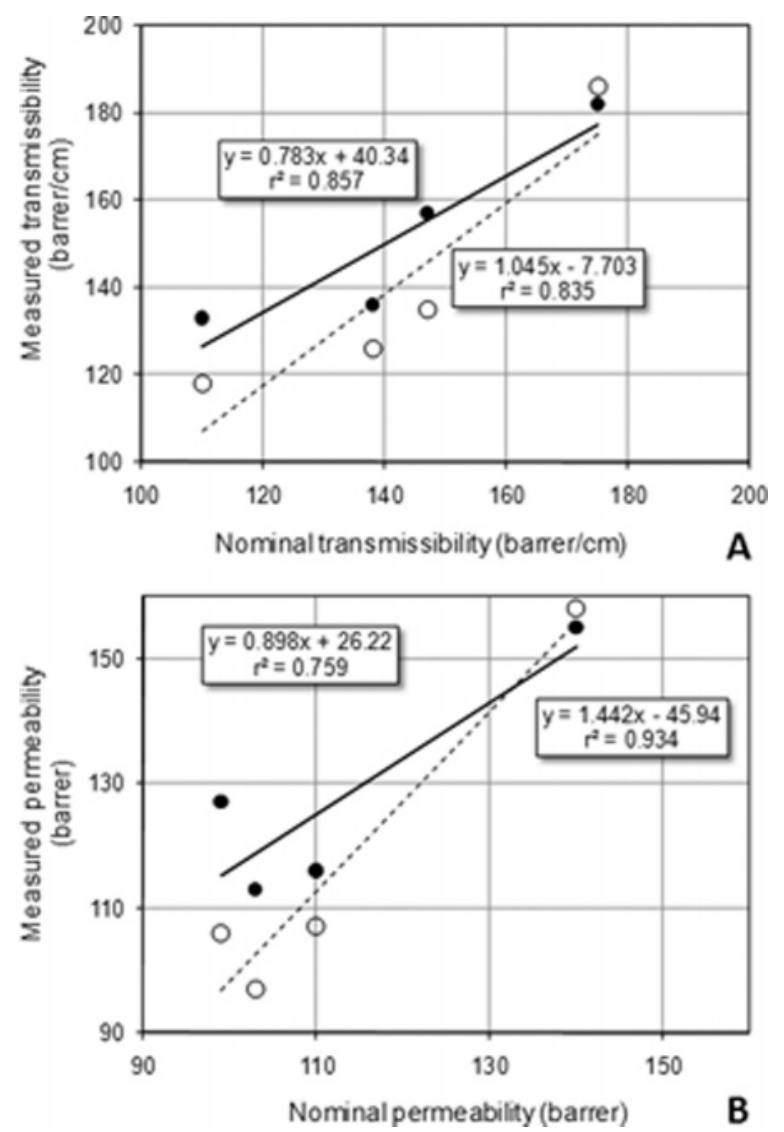

Figure 5. Regression of experimental $D k / t$ (A) and $D k$ values (B) derived from single samples of each material (filled circles) and the stack method (open circles) against the corresponding nominal values given by the manufacturers.

\section{Results}

Values of the resistance to the oxygen flow defined as $(D k /$ $t)^{-1}$ for each stack from $n=1-5$ CLs were plotted against the cumulated thickness in the stack. The plots for different materials, presented in Figure 4, show that the resistance of the stacks is a linear function of the thickness with correlation coefficients $r^{2} \geq 0.99$ for all of the $\mathrm{Si}-\mathrm{Hy}$ samples. However, some problems arise, as the plots of Figure 4 show. Actually, the plots are straight lines whose intercepts with the ordinate axis are slightly negative. This apparently senseless result can be explained by the uncertainty in the measurements of the apparent resistance of the stack indicated by bars and also by the extreme low values of the resistances of the layers flanking the stacks. Values of $(D k / t)_{\text {app }}$ and $D k$ obtained with this procedure, as well as $(D k / t)_{\text {app }}$ and $(D k)_{\text {app }}$ obtained from single sample and nominal values given by the manufacturers, are given in Tables 2 and 3, respectively.

The apparent oxygen transmissibility measured in this study for the five $\mathrm{Si}-\mathrm{Hy}$ materials ranged from $182 \pm 8 \mathrm{barrer} / \mathrm{cm}$ for lotrafilcon A to $133 \pm 5$ barrer/cm for balafilcon A, using the single sample method, and from 195 barrer for lotrafilcon A to 117 barrer for lotrafilcon B, using the stack method. In both cases, measured and nominal parameters were correlated $(p<0.001)$ although the results from both methods are slightly different (Figure 5). In general, values of $(D k / t)_{\text {app }}$ derived from single measurements are higher than those obtained from the stack method (Figure 6).

Table 4 presents the physiological values obtained by different methods and those reported by the manufacturers. Despite the differences observed in Tables 2 and 3 for $(D k / t)_{\text {app }}$ and $(D k)_{\text {app }}$
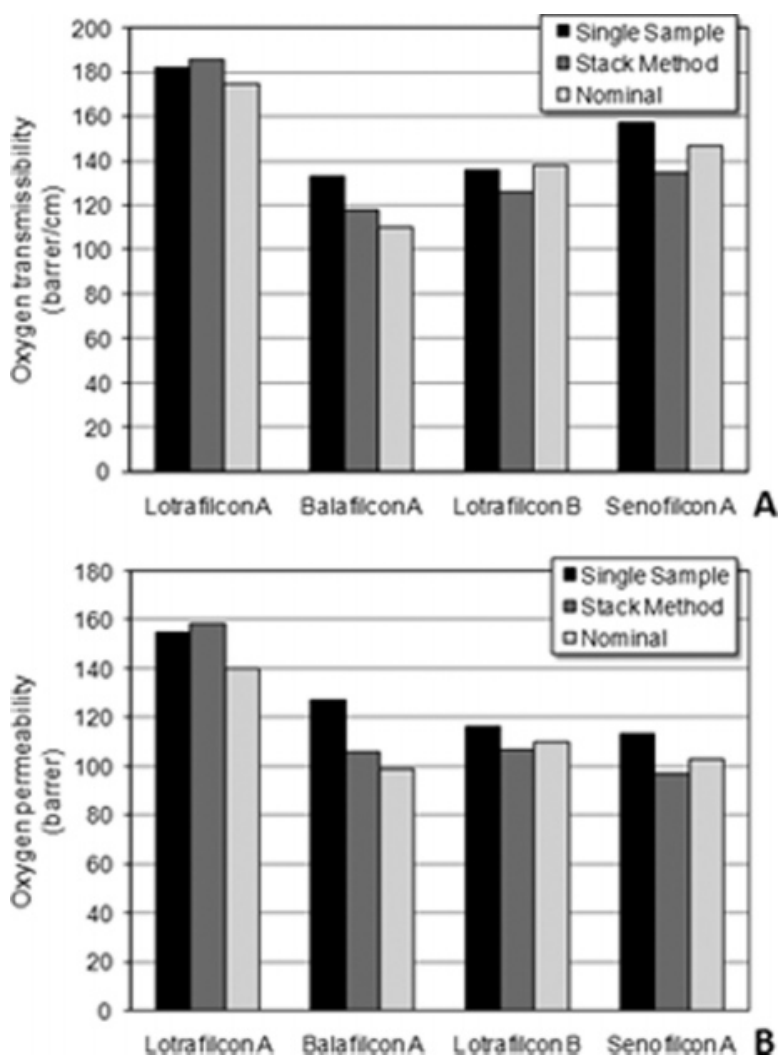

Figure 6. Comparison of the values of $D k / t$ (barrer/cm) and $D k$ (barrer) obtained by the two methods against the nominal values given by the manufacturer.

values, respectively, depending on the method used, no significant differences are observed in the results obtained for the physiological parameters calculated using the methods described. Despite some deviations in the values obtained by the two methods, and compared to the nominal results, the variation coefficient is lower than $2.8 \%$.

The partial pressure of oxygen at the cornea-CL interface $\left(p_{\mathrm{tc}}\right)$, oxygen flux $\left(j_{\mathrm{c}}\right), \mathrm{BOAT}$, and EOP are plotted as a function of the $\left(D k / t_{\mathrm{av}}\right)_{\text {app }}$ in Figures $7-10$. The $\left(D k / t_{\mathrm{av}}\right)_{\text {app }}$ window is 0-200 barrer/cm.

\section{Discussion}

With the advent of $\mathrm{Si}-\mathrm{Hy}$ materials, the question of oxygen permeability became a hot topic in the scientific literature. However, nowadays it is believed that oxygen transport should not be a matter of concern anymore because all current $\mathrm{Si}-\mathrm{Hy}$ materials warrant almost the same oxygenation levels to the eye. In fact, CLs made from hydrogels containing siloxane moieties in their structure present oxygen transmissibility 5-10 times higher than that reported for previously used extendedwear lenses made of conventional hydrogels. By conventional CLs, we mean hydrogels made of polymers not containing siloxane moieties in their structures, so that oxygen permeation mainly occurs through the water content of the swollen hydrogel. On the other hand, oxygen permeation across $\mathrm{Si}-\mathrm{Hy}$ takes place through siloxane-rich zones at substantially larger flow rates than those occurring in conventional hydrogels. ${ }^{10,30}$

The results obtained for the oxygen permeability in CLs using different measuring procedures significantly differ in some cases. Even with the same measurement method, remarkable differences could be expected. Morgan et al. ${ }^{34}$ found poor agreement between the results obtained by polarographic and coulometric techniques as well as between polarographic results and 
Table 4. Physiological Parameters Derived for Different CLs from Values of $D k / t_{\text {app }}$ Obtained by the Single Sample Method ${ }^{a}$, Stack Method ${ }^{b}$, and Nominal Values Given by the Manufacturers ${ }^{c}$, Respectively ${ }^{g}$

\begin{tabular}{|c|c|c|c|c|c|c|c|c|}
\hline & \multicolumn{2}{|c|}{ lotrafilcon A } & \multicolumn{2}{|c|}{ balafilcon A } & \multicolumn{2}{|c|}{ lotrafilcon B } & \multicolumn{2}{|c|}{ senofilcon A } \\
\hline & $143.6^{a}$ & & $139.5^{a}$ & & $139.8^{a}$ & & $141.8^{a}$ & \\
\hline \multirow[t]{3}{*}{$p_{\text {tc open }}(\mathrm{mmHg})$} & $143.8^{b}$ & $0.24^{f}$ & $137.6^{b}$ & $1.15^{f}$ & $138.7^{b}$ & $0.53^{f}$ & $139.7^{b}$ & $0.75^{f}$ \\
\hline & $143.1^{c}$ & & $136.4^{c}$ & & $140.0^{c}$ & & $140.9^{c}$ & \\
\hline & $46.1^{a}$ & & $43.1^{a}$ & & $43.3^{a}$ & & $44.8^{a}$ & \\
\hline \multirow[t]{3}{*}{$p_{\text {tc closed }}(\mathrm{mmHg})$} & $46.3^{b}$ & $0.57^{f}$ & $41.6^{b}$ & $2.82^{f}$ & $42.4^{b}$ & $1.28^{f}$ & $43.2^{b}$ & $1.77^{f}$ \\
\hline & $45.8^{c}$ & & $40.7^{c}$ & & $43.5^{c}$ & & $44.1^{c}$ & \\
\hline & $13.4^{a}$ & & $13.3^{a}$ & & $13.3^{a}$ & & $13.4^{a}$ & \\
\hline \multirow[t]{3}{*}{$\mathrm{BOAT}_{\text {open }}($ barrer/cm $)$} & $13.4^{b}$ & $0.10^{f}$ & $13.2^{b}$ & $0.28^{f}$ & $13.3^{b}$ & $0.13^{f}$ & $13.3^{b}$ & $0.19^{f}$ \\
\hline & $13.4^{c}$ & & $13.3^{c}$ & & $13.3^{c}$ & & $13.3^{c}$ & \\
\hline & $29.5^{a}$ & & $28.9^{a}$ & & $28.9^{a}$ & & $29.2^{a}$ & \\
\hline \multirow[t]{3}{*}{$\mathrm{BOAT}_{\text {closed }}($ barrer/cm) } & $29.5^{b}$ & $0.16^{f}$ & $28.7^{b}$ & $0.56^{f}$ & $28.8^{b}$ & $0.31^{f}$ & $28.9^{b}$ & $0.50^{f}$ \\
\hline & $29.4^{c}$ & & $28.6^{c}$ & & $29.0^{c}$ & & $29.1^{c}$ & \\
\hline & $19.4^{a}$ & & $18.8^{a}$ & & $18.9^{a}$ & & $19.1^{a}$ & \\
\hline \multirow[t]{3}{*}{$\mathrm{EOP}_{\text {open }}(\%)$} & $19.4^{b}$ & $0.24^{f}$ & $18.6^{b}$ & $1.15^{f}$ & $18.7^{b}$ & $0.53^{f}$ & $18.7^{b}$ & $0.75^{f}$ \\
\hline & $19.3^{c}$ & & $18.4^{c}$ & & $18.9^{c}$ & & $19.0^{c}$ & \\
\hline & $6.2^{a}$ & & $5.8^{a}$ & & $5.9^{a}$ & & $6.0^{a}$ & \\
\hline \multirow{3}{*}{$\mathrm{EOP}_{\text {closed }}(\%)$} & $6.3^{b}$ & $0.57^{f}$ & $5.6^{b}$ & $2.82^{f}$ & $5.7^{b}$ & $1.28^{f}$ & $5.8^{b}$ & $1.77^{f}$ \\
\hline & $6.2^{c}$ & & $5.5^{c}$ & & $5.9^{c}$ & & $6.0^{c}$ & \\
\hline & $20.8(7.5)^{a}$ & & $20.6(7.4)^{a}$ & & $20.6(7.4)^{a}$ & & $20.7(7.5)^{a}$ & \\
\hline \multirow[t]{3}{*}{ flux $_{\text {open }}$} & $20.8(7.5)^{b}$ & $0.10^{f}$ & $20.6(7.4)^{b}$ & $0.28^{f}$ & $20.6(7.4)^{b}$ & $0.13^{f}$ & $20.6(7.4)^{b}$ & $0.19^{f}$ \\
\hline & $20.8^{c}\left(7.5^{d}\right)$ & & $20.5^{c}\left(7.4^{d}\right)$ & & $20.6^{c}\left(7.4^{d}\right)$ & & $20.7^{d}\left(7.4^{e}\right)$ & \\
\hline & $16.2(5.8)^{a}$ & & $15.9(5.7)^{a}$ & & $15.9(5.7)^{a}$ & & $16.1(5.8)^{a}$ & \\
\hline \multirow[t]{2}{*}{ flux $_{\text {closed }}$} & $16.3(5.9)^{b}$ & $0.16^{f}$ & $15.8(5.7)^{b}$ & $0.56^{f}$ & $15.8(5.7)^{b}$ & $0.31^{f}$ & $15.9(5.7)^{b}$ & $0.50^{f}$ \\
\hline & $16.2^{c, d}\left(5.8^{e}\right)$ & & $15.7^{c, d}\left(5.7^{e}\right)$ & & $15.9^{c, d}\left(5.7^{e}\right)$ & & $16.0^{c, d}\left(5.8^{e}\right)$ & \\
\hline
\end{tabular}

${ }^{a}$ Derived from single measurement of $D k / t_{\text {app }}{ }^{b}$ Derived from the slope in the stack method. ${ }^{c}$ Derived from the slope in the stack method. ${ }^{d} 10^{-7} \cdot\left[\mathrm{cm}^{3} \mathrm{O}_{2}\left(S T_{\mathrm{p}}\right) \cdot \mathrm{cm}^{-2} \cdot \mathrm{s}^{-1}\right] .{ }^{e} \mu \mathrm{l}\left(\mathrm{O}_{2}\right) \cdot \mathrm{cm}^{-2} \cdot \mathrm{h}^{-1}$ barrer $\left.=10^{-11}\left(\mathrm{~cm}^{2} / \mathrm{sec}\right)\left[\mathrm{mL} \mathrm{O}_{2} /(\mathrm{mL} \times \mathrm{mmHg})\right]\right) .{ }^{f}$ Coefficient of variation $(\mathrm{CV} \%)$ of the three values as (SD/Mean)*100. CV for flux values is the same irrespective of the units. ${ }^{g}$ Partial pressure of oxygen at the CL-cornea interface $\left(p_{\mathrm{tc}}\right), \mathrm{BOAT}, \mathrm{EOP}(\%)$, and flux values for open- and closed-eye conditions were obtained from $(D k / t)_{\text {app }}$ determined from single and stack measurements. Nominal values provided by the manufacturer are included.

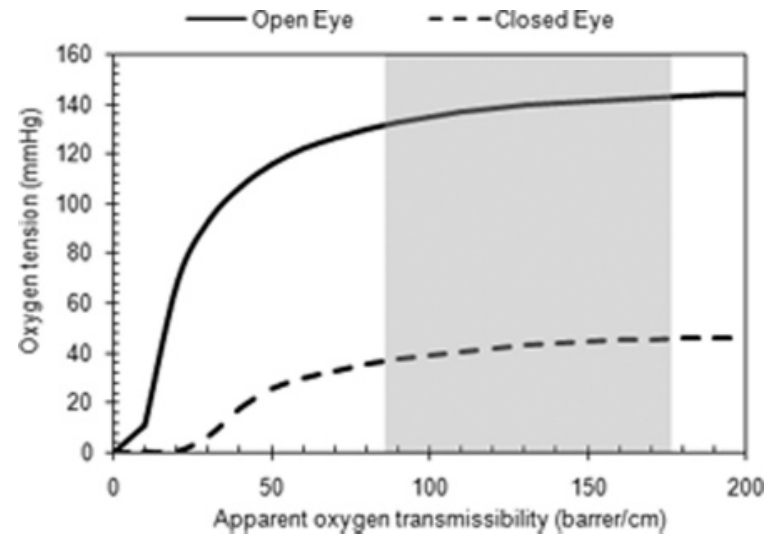

Figure 7. Oxygen tension $\left(p_{\mathrm{tc}}\right)$ at the cornea-CL interface under closed(solid line) and open-eyelid conditions (dashed line) for CLs of different transmissibility. The shaded area corresponds to the actual range of $D k / t$ for $\mathrm{Si}-\mathrm{Hy}$ materials.

reference values for RPG CLs as well as for the lotrafilcon A material. They explored different sources of error without finding a definitive explanation. Benjamin and The $D k$ Reference Study Group have recently assessed the oxygen permeability of the Permeability Reference Material Repository, consisting of 7 rigid gas permeable (RGP) materials with $D k$ values within the range of 10 to 161 barrers. In this study, the three different techniques currently available were used: the polarographic method corrected for boundary-layer and edge effects according to the American National Standards Institute Z80.20 standard (nine instruments), the coulometric method (four instruments), and the gas-to-gas method (two instruments). ${ }^{42}$ Small differences were found in the average values of both the apparent permeability and apparent transmissibility obtained for the same material in different laboratories using different experimental devices. The discrepancies persist when the measurements are performed with the same experimental device by different research groups in different laboratories.

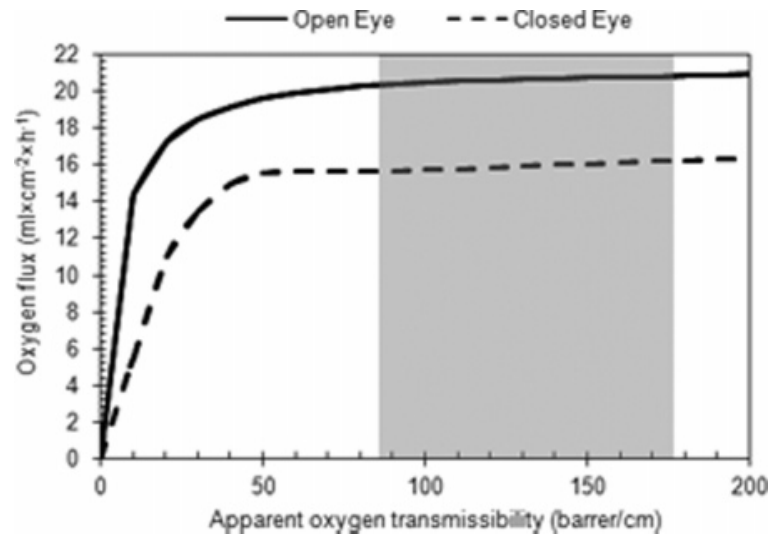

Figure 8. Oxygen flux $\left(j_{\mathrm{c}}\right)$ through CLs of different transmissibility for closed- (solid line) and open-eyelid conditions (dashed line). The shaded area corresponds to the actual range of $\mathrm{Dk} / \mathrm{t}$ for $\mathrm{Si}-\mathrm{Hy}$ materials.

Chhabra et al. ${ }^{36}$ found excellent agreement between the oxygen permeability of lens materials obtained by the singlelens method with that obtained using the conventional polarographic method utilized by us for conventional lenses, in the range from 9 to 180 barrers. The two methods used in this work, the single sample method and stack methods, render similar results in terms of correlation with nominal values for $D k / t$. However, the former method gave higher values of $D k / t$ than the latter; though if the errors indicated in Tables 2 and 3 are considered, our results are similar to those reported by the commercial lenses manufacturer. Conversely, when $D k$ values are required, the stack method showed a significantly better correlation with nominal values given by the manufacturer than those of the single sample method. Despite these differences, the values of the physiological parameters (partial pressure of oxygen at the $\mathrm{CL}$-cornea interface $\left(p_{\mathrm{tc}}\right), \mathrm{BOAT}, \mathrm{EOP}$, and oxygen flux $\left(j_{\mathrm{c}}\right)$ ) do not change significantly for open- and closed-eye conditions for the materials evaluated in this work. This fact is better explained by the simulations given in Figures 


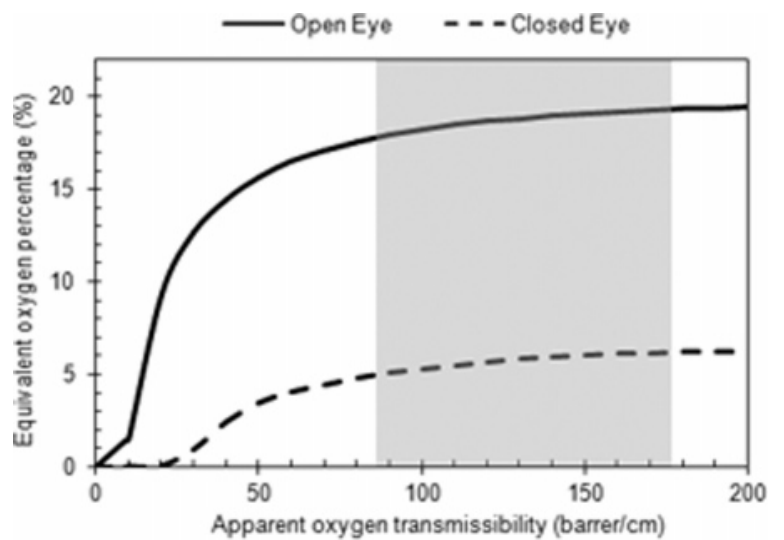

Figure 9. EOP available for CLs of different transmissibility under closed(solid line) and open-eyelid conditions (dashed line). The shaded area corresponds to the actual range of $\mathrm{Dk} / \mathrm{t}$ for $\mathrm{Si}-\mathrm{Hy}$ materials.

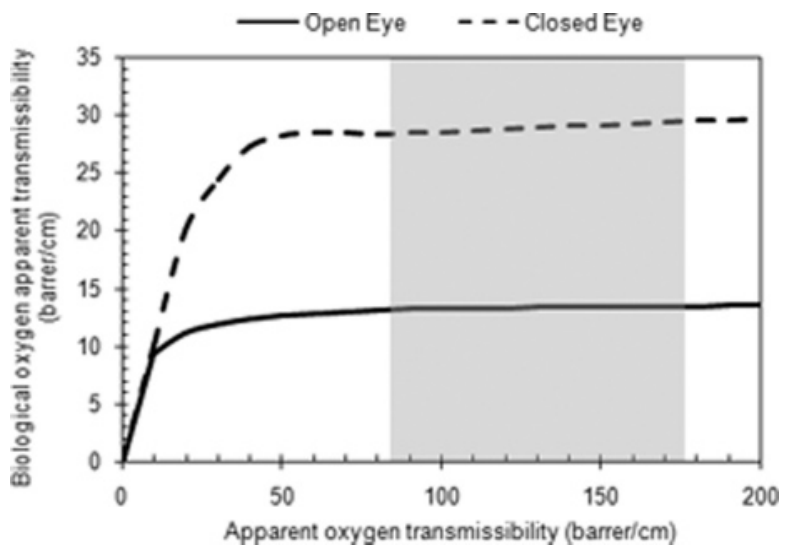

Figure 10. BOAT for CLs of different transmissibility under closed- (solid line) and open-eyelid conditions (dashed line). The shaded area corresponds to the actual range of $D k / t$ for $\mathrm{Si}-\mathrm{Hy}$ materials.

7-10, which show that the values of all of the physiological parameters do not vary substantially with changes in the values of $D k / t$. As a consequence, a single $D k / t$ measurement would be acceptable to determine the physiological environment for a given $\mathrm{Si}-\mathrm{Hy} \mathrm{CL}$ material with IOT coefficients lying in the range of 110-175 barrers. However, a single sample measurement is not acceptable for the estimation of EOP and $p_{\mathrm{tc}}$ if the IOT is lower than $70-100 \mathrm{barrer} / \mathrm{cm}$. The method is neither suitable to determine the oxygen flow and the BOAT in lenses where: (a) the transmissibility is below 50 barrers, (b) $D k / t_{\mathrm{av}}$ is lower than 70-100 barrers/cm if EOP is to be determined, and (c) $D k / t_{\mathrm{av}}$ is below 50 barrers if the oxygen flux and BOAT are to be computed.

Some of the results obtained by us with the polarographic method are in agreement with those reported by Chhabra et al. ${ }^{36}$ $(108 \pm 5$ and $181 \pm 17$ barrer/cm for Purevision and Focus N\&D, respectively) using a novel polarographic apparatus. Also, by using the liquid-to-gas arrangement in the coulometric device, Morgan et al. ${ }^{34}$ and Alvord et al. ${ }^{35}$ found similar values for the $D k$ of lotrafilcon, specifically, $150 \pm 4$ and $155 \pm 5$ barrer, respectively. The extensive work carried out by Compañ et al. ${ }^{10,30}$ in recent years with the first generation of $\mathrm{Si}-\mathrm{Hy}$ materials using the stack method and a polarographic device also support the values obtained in the present work. Compañ et al. ${ }^{30}$ obtained $D k$ values of $141 \pm 5$ and $107 \pm 4$ barrers for lotrafilcon A and balafilcon A materials using the stack procedure and a polarographic sensor. These values yield $(D k /$ $t)_{\text {app }}$ equal to $183 \pm 7$ and $123 \pm 5$ barrers/cm, respectively.

The simulated relationships between $(D k / t)_{\text {app }}$ and the physiological variables involving partial pressure at the $\mathrm{CL}$-cornea interface, BOAT, and EOP (or oxygen flux to the cornea) might assist clinicians to evaluate the conformity of the lenses they are fitting with the requirements of the cornea. For example, for open-eyelid conditions, lenses with $D k / t$ values below 30 barrer/cm will only supply $60 \%$ of the partial pressure of oxygen provided by the ideal CL of infinite $D k / t_{\mathrm{av}}$. This fact supports early estimations of Holden and Mertz ${ }^{8}$ indicating that at least 34 barrers/cm would be necessary to avoid corneal hypoxia under open-eyelid conditions. For closed-eyelid conditions, lenses with less than $80 \mathrm{barrer} / \mathrm{cm}$ only provide about $60 \%$ of the partial pressure of oxygen provided by an ideal CL. Holden and Mertz's ${ }^{8}$ criteria stating that a $D k / t_{\text {av }}$ of at least 87 barrer/ $\mathrm{cm}$ is required to limit the corneal edema to physiologic levels during overnight CL wear only warrant about $65 \%$ of the ideal partial pressure at the CL corneal interface. Thus, it makes sense to change the requirements to those stated by Harvitt and Bonnano. ${ }^{9}$ According to these requirements, a minimum of 125 barrers/cm are necessary for closed-eyelid conditions. This transmissibility guarantees about $75 \%$ of the ideal partial pressure of oxygen at the cornea-CL interface.

At first sight, the BOAT - $D k / t_{\text {av }}$ relationship seems to be surprising as BOAT values are higher for closed-eyelid than for open-eyelid conditions, a fact particularly evident for $D k$ / $t_{\mathrm{av}}$ values above 10 barrer/cm. However, this can be explained considering the definition of the BOAT as a representative parameter of the potential of diffusion of oxygen through lenses. According to Fick's first law, oxygen transport across lenses will be higher as both transmissibility and oxygen concentration gradient increase. Thus, under closed-eyelid conditions, the low partial pressure of oxygen at the CL-cornea interface increases the BOAT parameter. Another observation is that for values of $D k / t$ below 20-25 barrers/cm, BOAT and $D k / t$ follow a $1: 1$ relationship for closed-eyelid conditions. Above a certain level of transmissibility $(20-30$ barrer/cm for open-eyelid and 50 barrer/cm for closed-eyelid conditions), a further increase in $D k / t$ will not result in a significant positive reflection in the potential of diffusion of oxygen through the lens. This is because the partial pressure of oxygen at the cornea-CL interface increases significantly, thus decreasing the gradient of partial pressure (previously described as relative variation of the partial pressure of oxygen between the front $(p)$ and the back sides $\left(p_{\mathrm{tc}}\right)$ of a lens onto the cornea). Any effect of the stagnation of oxygen at the cornea-CL interface (oxygen not consumed by the cornea) does not conduct to the augment of the BOAT because the difference between $p$ and $p_{\text {tc }}$ decreases as $p_{\text {tc }}$ increases.

Both lotrafilcon A and senofilcon A CLs meet the more recent criteria for avoiding stromal edema during overnight wear. The high oxygen transmissibility of $\mathrm{Si}-\mathrm{Hy}$ materials has provided substantial improvements in corneal physiology with almost no evidence of edema under overnight wear, ${ }^{43}$ and even allows patients with active conditions in the ocular surface to wear them for therapeutic purposes. ${ }^{18-21,44}$ Other situations that can potentially compromise ocular physiology as combined fitting of a RGP CL piggybacked over a soft CL have experienced remarkable improvements in terms of oxygen performance when highly permeable $\mathrm{Si}-\mathrm{Hy}$ materials are used. ${ }^{45-47}$

\section{Conclusions}

The values of $(D k / t)$ app and $D k$ can vary significantly depending on the method of measurement, though the physiological values that are relevant to evaluate the physiological performance of CLs do not undergo significant changes. Thus, in the range of high transmissibilities $(110-175$ barrer/cm), significant variations in the values of $D k / t$ have low impact on 


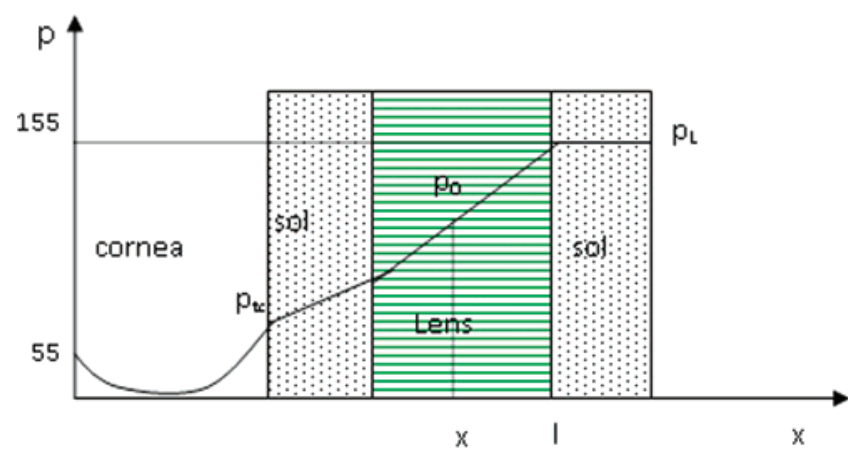

Figure 11. Pressure gradient in the cornea-lens system in the stationary state conditions in $\mathrm{mmHg}$. The oxygen partial pressure at the entire outer surface of the CL and the endothelium/aqueous humor interface are 155 and $55 \mathrm{mmHg}$, respectively.

the physiological performance of the lenses, and the results calculated from single samples do not differ significantly from those obtained in the stacked method. However, these assumptions are not true for low $(D k / t)$ app values (i.e., below 70 barrer/ $\mathrm{cm})$. So, for material characterization and accurate measurements of oxygen transmissibility and permeability, the stack method should be used, although some variability in the results can be expected for materials with high $(D k / t)_{\text {app }}$. On view of the significant variability of current techniques to measure material properties, more accurate and repeatable methods are necessary to measure these properties for CL materials. However, these differences are not likely to remain significant when translated into physiological variables of partial pressure at the $\mathrm{CL}$ - cornea interface, BOAT, EOP, or oxygen flux to the cornea. The same would not apply to other CLs whose $\mathrm{Dk} / \mathrm{t}$ lies below 50-100 barrer/cm.

\section{Appendix A}

The non-steady-state diffusion equation that gives oxygen tension as a function of time and position for a homogeneous slab of oxygen-consuming tissue (as a cornea in a 1D model) is

$$
\frac{\partial^{2} p_{\mathrm{c}}}{\partial x^{2}}-\frac{Q}{D k}=\frac{\partial p_{\mathrm{c}}}{\partial t}
$$

where $D$ is the diffusion coefficient of oxygen in the tissue, $p_{\mathrm{c}}$ is the partial pressure of oxygen in the cornea, $k$ is Henry's law constant, $Q$ is the consumption rate, $x$ the distance perpendicular to the surface, and $t$ is time. For the steady-state case, eq A.1 becomes

$$
\frac{d^{2} p_{\mathrm{c}}}{\mathrm{d} x^{2}}=\frac{Q}{D k}
$$

At steady-state conditions, the following expression holds at the lens-cornea interface

$$
\frac{d^{2} p_{\mathrm{tc}}}{d x^{2}}=0
$$

Figure 11 shows the pressure in the cornea-lens interface, in steady-state conditions, supposing that in the endothelium the oxygen partial pressure is $55 \mathrm{mmHg}$.

The solutions of eqs A.2 and A.3 are

$$
\begin{gathered}
p_{\mathrm{c}}=A x^{2}+B x+C \\
p_{\mathrm{tc}}=B^{\prime} x+C^{\prime}
\end{gathered}
$$

where the constants $A, B, C, B^{\prime}$, and $C^{\prime}$ are determined as follows. The constant $A$ can be obtained by equating the second derivative of eq A.4 to eq A.2, giving

$$
A=\frac{Q}{2 D k}
$$

The constant $C$ can be determined by taking as origin of coordinates $(x=0)$ of the interface of the posterior part of the cornea (endothelium) in contact with the aqueous humor. Assuming that the partial pressure in this interface is the same as that on the cornea surface $(55 \mathrm{mmHg})$, then

$$
p_{\mathrm{c}}=C
$$

The partial pressure of oxygen on the surface of the closed eyes, $55 \mathrm{mmHg}$, is taken as the minimum pressure necessary to prevent corneal swelling above $4 \%$. It can be further assumed that $p_{\mathrm{c}} \cong p_{\mathrm{tc}}$ due to the small thickness and high transmissibility of the tear film, in comparison with those of the cornea. Hence,

$$
A t_{\mathrm{c}}^{2}+B t_{\mathrm{c}}+C=B^{\prime} t_{\mathrm{av}}+C^{\prime}
$$

where $t_{\mathrm{c}}$ is the cornea thickness. Eqs A.4 through eq A.7 yield

$$
\frac{Q}{2 D k} t_{\mathrm{c}}^{2}+B t_{\mathrm{c}}+55=B^{\prime} t_{\mathrm{av}}+C^{\prime}
$$

Because the partial pressures of oxygen in the open- and closed-eyelids situations are, respectively, 155 and $55 \mathrm{mmHg}$, eq A.5 can be written as

$$
\begin{gathered}
B^{\prime} t^{\prime}+C^{\prime}=155 \\
B^{\prime} t^{\prime}+C^{\prime}=55
\end{gathered}
$$

where $t^{\prime}=t_{\mathrm{c}}+t_{\mathrm{av}}$ because, as indicated above, the origin of the coordinates' reference frame is assumed to be located at the interface of the endothelium with the aqueous humor. ${ }^{40} \mathrm{At}$ the lens-cornea interface,

$$
j_{\mathrm{c}}=j_{\mathrm{tc}}
$$

so that

$$
(D k)_{\mathrm{c}}\left(\frac{\mathrm{d} p}{\mathrm{~d} x}\right)_{\mathrm{c}}=(D k)_{\mathrm{tc}}\left(\frac{\mathrm{d} p}{\mathrm{~d} x}\right)_{\mathrm{tc}}
$$

By substituting eqs A.4 and A.5 into eq A.12, we obtain

$$
(D k)_{\mathrm{c}}\left[\frac{Q t}{D k}+B\right]_{\mathrm{c}}=(D k)_{\mathrm{tc}} B^{\prime}
$$

where the value of $A$ given by eq A.6 was considered.

By combining eqs A.9 to A.13, a system of three equations with three unknown parameters $\left(B, B^{\prime}\right.$, and $\left.C^{\prime}\right)$, it is obtained

$$
\begin{gathered}
t_{\mathrm{c}} B-t_{\mathrm{av}} B^{\prime}-C^{\prime}=-\left(\frac{Q}{2 D k}\right)_{\mathrm{c}} t_{\mathrm{c}}^{2}-55 \\
(D k)_{\mathrm{c}} B-(D k)_{\mathrm{tc}} B^{\prime}=-Q t_{\mathrm{c}} \\
t^{\prime} B^{\prime}+C^{\prime}=155
\end{gathered}
$$


This system permits us to determine the partial pressure of oxygen at the lens-cornea interface, $p_{\text {tc }}$. For the closed-eye situation, eq A.10-b is substituted with eq A.14-c.

Using the thickness, $t_{\mathrm{av}}$, and the values of the apparent permeability coefficients, $(D k)_{\mathrm{tc}}$, given in Table 1 , the three cornea parameters reported by Fatt and Weissman ${ }^{40}$ (cornea thickness: $t_{\mathrm{c}}=0.05 \mathrm{~cm}$, cornea oxygen permeability coefficient: $(D k)_{c}=24.7$ barrers $\left(1\right.$ barrer $=10^{-11} \times\left[\mathrm{cm}^{3} \mathrm{O}_{2}\left(S T_{\mathrm{p}}\right)^{\cdot} \cdot \mathrm{cm}^{\bullet} \mathrm{cm}^{-2}\right.$ $\left.\mathrm{s}^{-1}(\mathrm{mmHg})^{-1}\right]$, as well as the flow of oxygen onto the cornea $\left(Q \cong 6.610^{-5} \mathrm{~cm}^{3} \mathrm{O}_{2}\left(S T_{\mathrm{p}}\right) \cdot \mathrm{cm}^{-3} \cdot \mathrm{s}^{-1}\right)$, the values of $B, B^{\prime}$, and $C^{\prime}$ were obtained. These values, in conjunction with eq A.4, allow us to determine $p_{\text {tc. }}$.

\section{Acknowledgment}

We would like to acknowledge financial support from the IMPIVA. This work, supported by the Dirección General de Investigación Científica y Técnica (DGICYT), grant MAT-200505648-C02-01, is gratefully acknowledged. We also acknowledge Generalitat Valenciana through Instituto de la Pequeña y Mediana Industria Valenciana (IMPIVA), Grant IMCOVA$2006 / 20$. This work was also supported in part by a grant from the Science and Technology Foundation (FCT) of the Portuguese Ministry of Science and Superior Education and through the European Social Funding (ESF) under contract \#8281/2002 granted to J.M.G.-M.

\section{Literature Cited}

(1) Erickson, P.; Comstock, T. L.; Zantos, S. G. Effects of Hydrogel Lens Transmissibility Profiles on Local Corneal Swelling During Eye Closure. Optom. Vis. Sci. 1996, 73, 169-177.

(2) Papas, E. B. The Role of Hypoxia in the Limbal Vascular Response to Soft Contact Lens Wear. Eye Contact Lens 2003, 29, S72-S74.

(3) Perez, J. G.; Meijome, J. M.; Jalbert, I., et al. Corneal Epithelial Thinning Profile Induced by Long-Term Wear of Hydrogel Lenses. Cornea 2003, 22, 304-307.

(4) Holden, B. A.; Sweeney, D. F.; Vannas, A., et al. Effects of LongTerm Extended Contact Lens Wear on the Human Cornea. Invest. Ophthalmol. Vis. Sci. 1985, 26, 1489-1501.

(5) Keay, L.; Sweeney, D. F.; Jalbert, I., et al. Microcyst Response to High Dk/t Silicone Hydrogel Contact Lenses. Optom. Vis. Sci. 2000, 77, $582-585$.

(6) Hill, R. M.; Fatt, I. Oxygen Uptake from a Reservoir of Limited Volume by the Human Cornea In Vivo. Science 1963, 142, 1295-1297.

(7) Brennan, N. A. A Model of Oxygen Flux through Contact Lenses. Cornea 2001, 20, 104-108.

(8) Holden, B. A.; Mertz, G. W. Critical Oxygen Levels to Avoid Corneal Edema for Daily and Extended Wear Contact Lenses. Invest. Ophthalmol. Vis. Sci. 1984, 25, 1161-1167.

(9) Harvitt, D. M.; Bonanno, J. A. Re-evaluation of the Oxygen Diffusion Model for Predicting Minimum Contact Lens Dk/t Values Needed to Avoid Corneal Anoxia. Optom. Vis. Sci. 1999, 76, 712-719.

(10) Compañ, V.; Lopez-Alemany, A.; Riande, E.; Refojo, M. F. Biological Oxygen Apparent Transmissibility of Hydrogel Contact Lenses with and without Organosilicon Moieties. Biomaterials 2004, 25, 359365 .

(11) Brennan, N. A. Beyond Flux: Total Corneal Oxygen Consumption as an Index of Corneal Oxygenation during Contact Lens Wear. Optom. Vis. Sci. 2005, 82, 467-472.

(12) Aakre, B. M.; Ystenaes, A. E.; Doughty, M. J., et al. A 6-Month Follow-Up of Successful Refits from Daily Disposable Soft Contact Lenses to Continuous Wear of High-Dk Silicone-Hydrogel Lenses. Ophthalmic. Physiol. Opt. 2004, 24, 130-141.

(13) Brennan, N. A.; Coles, M. L.; Comstock, T. L.; Levy, B. A 1-Year Prospective Clinical Trial of Balafilcon a (PureVision) Silicone-Hydrogel Contact Lenses Used on a 30-Day Continuous Wear Schedule. Ophthalmology 2002, 109, 1172-1177.

(14) Gonzalez-Meijome, J. M.; Gonzalez-Perez, J.; Cervino, A., et al. Changes in Corneal Structure with Continuous Wear of High-Dk Soft Contact Lenses: A Pilot Study. Optom. Vis. Sci. 2003, 80, 440-446.

(15) Maldonado-Codina, C.; Morgan, P. B.; Efron, N.; Efron, S. Comparative Clinical Performance of Rigid versus Soft Hyper Dk Contact Lenses Used for Continuous Wear. Optom. Vis. Sci. 2005, 82, 536-548.
(16) McNally, J. J.; Chalmers, R. L.; McKenney, C. D.; Robirds, S. Risk Factors for Corneal Infiltrative Events with 30-Night Continuous Wear of Silicone Hydrogel Lenses. Eye Contact Lens 2003, 29, S153-S156.

(17) Morgan, P. B.; Efron, N. Comparative Clinical Performance of Two Silicone Hydrogel Contact Lenses for Continuous Wear. Clin. Exp. Optom. 2002, 85, 183-192.

(18) Ambroziak, A. M.; Szaflik, J. P.; Szaflik, J. Therapeutic Use of a Silicone Hydrogel Contact Lens in Selected Clinical Cases. Eye Contact Lens 2004, 30, 63-67.

(19) Kanpolat, A.; Ucakhan, O. O. Therapeutic Use of Focus Night \& Day Contact Lenses. Cornea 2003, 22, 726-734.

(20) Montero, J.; Sparholt, J.; Mely, R. Retrospective Case Series of Therapeutic Applications of a Lotrafilcon A Silicone Hydrogel Soft Contact Lens. Eye Contact Lens 2003, 29, S54-S56.

(21) Szaflik, J. P.; Ambroziak, A. M.; Szaflik, J. Therapeutic Use of a Lotrafilcon A Silicone Hydrogel Soft Contact Lens as a Bandage After LASEK Surgery. Eye Contact Lens 2004, 30, 59-62.

(22) Fatt, I.; Chaston, J. Measurement of Oxygen Transmissibility and Permeability of Hydrogel Lenses and Materials. Int. Contact Lens Clin. 1982, 9, 76-88.

(23) Refojo, M. F.; Holly, F. J.; Leong, F. L. Permeability of Dissolved Oxygen through Contact Lenses I. Cellulose Acetate Butyrate. Contact Intraocular Lens Medical J. 1977, 3, 27-32.

(24) Refojo, M. F.; Leong, F. L. Water-Dissolved-Oxygen Permeability Coefficients of Hydrogel Contact-Lenses and Boundary-Layer Effects. $J$. Membr. Sci. 1979, 4, 415-426.

(25) Brennan, N.; Efron, N.; Holden, B. A. Oxygen Permeability of Hard Gas Permeable Contact Lens Materials. Clin. Exp. Optom. 1986, 69, 8289.

(26) Compañ, V.; Villar, M. A.; Valles, E.; Riande, E. Permeability and Diffusional Studies on Silicone Polymer Networks with Controlled Dangling Chains. Polymer 1996, 37, 101-107.

(27) Paterson, R.; Doran, P. A Spray Technique for the Determination of Membrane Diffusion and Distribution Coefficients by the Time-Lag Method: Evaluated for Electrolyte Transport through Charged and Uncharged Membranes. J. Membr. Sci. 1986, 26, 289-301.

(28) Winterton, L.; White, J.; Su, K. Coulometric Method for Measuring Oxygen Flux and Dk of Contact Lenses and Lens Materials. Int. Contact Lens Clin. 1987, 14, 441-451.

(29) Aiba, S.; Ohashi, M.; Huang, S. Rapid Determination of Oxygen Permeability of Polymer Membranes. Ind. Eng. Chem. Fundam. 1968, 7 , 497-502.

(30) Compañ, V.; Andrio, A.; Lopez-Alemany, A., et al. Oxygen Permeability of Hydrogel Contact Lenses with Organosilicon Moieties. Biomaterials 2002, 23, 2767-2772.

(31) Young, M. D.; Benjamin, W. J. Oxygen Permeability of the Hypertransmissible Contact Lenses. Eye Contact Lens 2003, 29, S17-S21.

(32) Benjamin, W. J. Oxygen Transport through Contact Lenses. In Contact Lens Practice; Ruben, M., Guillon, M., Eds.; Chapman \& Hall: London, 1994; pp 43-70.

(33) ISO International Standard 9913-1. Contact Lenses: Part 1: Determination of Oxygen Permeability and Transmissibility by the FATT Method. In International Standards Organization, (ISO) ed. International Standard, Optics and Optical Instruments; Case Postale 56, CH-1211: Geneva, Switzerland, 1996; pp 1-13.

(34) Morgan, C. F.; Brennan, N. A.; Alvord, L. Comparison of the Coulometric and Polarographic Measurement of a High-Dk Hydrogel. Optom. Vis. Sci. 2001, 78, 19-29.

(35) Alvord, L.; Court, J.; Davis, T., et al. Oxygen Permeability of a New Type of High Dk Soft Contact Lens Material. Optom. Vis. Sci. 1998, $75,30-36$

(36) Chhabra, M.; Prausnitz, J. M.; Radke, C. J. A Single-Lens Polarographic Measurement of Oxygen Permeability (Dk) for Hypertransmissible Soft Contact Lenses. Biomaterials 2007, 28, 4331-4342.

(37) Goldenberg, M. S.; Rennwantz, E.; Beekman, A. Polarographic Evaluation of Highly Oxygen Permeable Silicon Rubber: Correction for Electrode Nonlinearity and Implications for Contact Lenses. Int. Contact Lens Clin. 1991, 18, 154-161.

(38) Compañ, V.; López, M.; Andrio, A., et al. Determination of the Oxygen Transmissibility and Permeability of Hydrogel Contact Lenses. $J$. Appl. Polym. Sci. 1999, 72, 321-327.

(39) Fatt, I.; Ruben, C. M. New Oxygen Transmissibility Concept for Hydrogel Contact Lenses. J. Br. Contact Lens Assoc. 1993, 16, 141-149.

(40) Fatt, I.; Weissman, B. A. Physiology of the Eyes, 2nd ed.; Butterworth-Heinemann: Boston; 1992; p 161.

(41) Turnbull, D. K.; Gathiram, P.; Bhagwan, I., et al. Oxygen Flux through Dry and Wet Hard Gas-Permeable Contact Lenses. J. Brit. Cont. Lens Assoc. 1986, 9, 75-84. 
(42) Benjamin, W. J. The Dk Reference Study Group. Revised Oxygen Permeability (Dk) of Reference Materials. Invest. Ophthalmol. Vis. Sci. 2006, 47, 97.

(43) Steffen, R. B.; Schnider, C. M. The Impact of Silicone Hydrogel Materials on Overnight Corneal Swelling. Eye Contact Lens 2007, 33, $115-$ 120.

(44) Ozkurt, Y.; Rodop, O.; Oral, Y., et al. Therapeutic Applications of Lotrafilcon A Silicone Hydrogel Soft Contact Lenses. Eye Contact Lens 2005, 31, 268-269.

(45) Lopez-Alemany, A.; Gonzalez-Meijome, J. M.; Almeida, J. B., et al. Oxygen Transmissibility of Piggyback Systems with Conventional Soft and Silicone Hydrogel Contact Lenses. Cornea 2006, 25, 214-219.
(46) O'Donnell, C.; Maldonado-Codina, C. A Hyper-Dk Piggyback Contact Lens System for Keratoconus. Eye Contact Lens 2004, 30, 4448.

(47) Florkey, L. N.; Fink, B. A.; Mitchell, G. L.; Hill, R. M. Corneal Oxygen Uptake Associated with Piggyback Contact Lens Systems. Cornea 2007, 26, 324-335.

Received for review October 17, 2007 Revised manuscript received February 5, 2008 Accepted February 6, 2008

IE071403B 